Hongwei et al., Afr J Tradit Complement Altern Med., (2018) 15 (2): 111-117

https://doi.org/10.21010/ajtcam.v15i2.14

\title{
EFFECT OF KANGFUXIN LIQUID ON INTESTINAL BARRIER IN ELDERLY PATIENTS WITH MECHANICAL VENTILATION IN THE INTENSIVE CARE UNIT
}

\section{Zhang Hongwei $^{1}$, Wei Liyou ${ }^{2}{ }^{*}$, Zhao Gang ${ }^{1}$, Liu Shuzheng ${ }^{1}$, Zhang Jing ${ }^{3}$}

${ }^{1}$ Intensive Care Unit, The second Hospital of Tangshan, Tangshan, China 063000; ${ }^{2}$ Orthopaedics, The second Hospital of Tangshan, Tangshan, China 063000; ${ }^{3}$ Intensive Care Unit, The people's Hospital of Tangshan, Tangshan, China 063000.

*Corresponding author's E-mail: weiliyou888@163.com

\begin{tabular}{|l|}
\hline \multicolumn{1}{|c|}{ Article History } \\
Received: Sept. 23, 2016 \\
Revised Received: Dec. 28, 2017 \\
Accepted: Dec. 28, 2017 \\
Published Online: Feb. 23, 2018. \\
\hline
\end{tabular}

\begin{abstract}
Background: Kangfuxin Liquid is extracted from America periplaneta, which is effective for treating gastrointestinal ulcers and repairing gastrointestinal mucosal tissue. The purpose of this study is to explore the effect of Kangfuxin Liquid on improving intestinal barrier dysfunction in elderly patients using mechanical ventilation and effectively restraining the concentration of D-lactate.
\end{abstract}

Materials and Methods: Elderly patients with mechanical ventilation were selected, and divided into "control" and "treatment" groups by using a random number table. Patients in both groups were given conventional treatment. Patients in "treatment group" additionally received Kangfuxin Liquid. At baseline and at 3, 7days following treatment, recordings for the concentration of D-lactate, intra-abdominal pressure (IAP) and bowel sounds were conducted. The duration of mechanical ventilation, the time of stay in ICU and the mortality rate during 28 days were recorded.

Results: At baseline, there were no significant differences in the terms of the concentrations of D-lactate, IAP and bowel sounds in the two groups ( $\mathrm{P}>0.05)$. At 3 and 7 days following treatment, the concentration of D-lactate, IAP and bowel sounds in the "treatment group" were better than those in the "control group". The differences were statistically significant $(\mathrm{P}<0.05)$. The duration of mechanical ventilation and the time of stay in intensive care unit (ICU) in the treatment group were less than those in the control group $(\mathrm{P}<0.05)$.

Conclusion: Kangfuxin Liquid can restrain the concentration of D-lactate, improves intestinal mucosal permeability, enhances the intestinal barrier, and improves the condition in elderly patients with mechanical ventilation.

Keywords: Kangfuxin Liquid, The elderly, Intestinal barrier, D- lactate.

Abbreviations: IAP, intra-abdominal pressure; ICU, intensive care unit; SIRS, systemic inflammatory response syndrome; MODS, multiple organ dysfunction syndrome; IgA, immunoglobulin A ; IgG, immunoglobulin G.

\section{Introduction}

During recent years, elderly patients with mechanical ventilation are gradually rising as a result of population demographics. The reserve of intestinal barrier function in the elderly patient declines, intestinal vascular shrink under the condition of mechanical ventilation, accompanied by ischemia and hypoxia of mucosal tissue. Consequently 
intestinal barrier dysfunction and worse yet- failure often occurs (Pan et al., 2016). When elderly patients have intestinal barrier dysfunction, it can be argued that this condition had been aggravated or alternatively that the prognosis was poor (Geboes et al., 2002). Therefore, it has practical significance to protect elderly patients who have intestinal barrier function with mechanical ventilation in the early stage.

At present, the topic of elderly patients in critical stages with intestinal barrier dysfunction has been receiving more attention and likewise its prevention. Many traditional medicines for treating intestinal barrier dysfunction have achieved satisfactory results (Qiu et al., 2011; Fang et al., 2006). Kangfuxin Liquid is extracted from America periplaneta, which belongs to the insect medicines with effectiveness on gastrointestinal ulcer and repairs gastrointestinal mucosal tissue (Wang et al., 2013; Zhang et al., 2012). Kangfuxin Liquid is used to improve the intestinal barrier dysfunction in elderly patients, and explore the effect on restraining the concentration of D-lactate.

\section{Materials and Methods}

Trial design: This study used a prospective randomized controlled trial and the sample size was first established and evaluated. The subject patients were assigned into "treatment" and "control" groups using a random number table with thirty-one patients comprising each group. The detection and record of evaluation index was performed by two independent investigators. Both investigators were unaware of the treatment allocation.

Study population: During the period January 2010 to December 2012, a total of 62 elderly patients with mechanical ventilation in ICU were selected from our hospitals, 35 male, 27 female, ranging in age from 61 to 75 years. Their primary diseases were severe pneumonia, pulmonary embolism, chest trauma, cerebro-vascular disease, brain injury, poisoning, and other illnesses.

Ethics: This research protocol was approved by our hospital's committee in accordance with the ethical standards set out in the 1964 Declaration of Helsinki and its later amendments. Before commencing this study all patients or their families were informed, consent given for treatment, and an agreement was signed. .

Inclusion criteria: The patients with mechanical ventilation were older than 60 years of age; Their APACHE II score was greater than or equal to 12 points; and their records indicated they never had peptic ulcers or any other gastrointestinal disease.

Exclusion criteria: Patients not chosen were those who had the following issues: Patients who had used anti cholinergic or gastrointestinal motility medicines within the previous week, those suffering from primary disease in their digestive system, others with severe intra- abdominal infections, those infected with human immunodeficiency virus, and those with Parkinson's disease, hyperthyroidism and epilepsy.

Intervention: All patients were provided comprehensive treatment, including the treatment of their initial disease, blood transfusion, oxygen, analgesic sedation, maintenance of water electrolyte balance and any other considerations. There were no statistically differences between these two sample groups. "Treatment" patients additionally received Kangfuxin Liquid (Hunan Kelun Pharmaceutical Co., Ltd. Chian), Kangfuxin Liquid via a naso-gastric tube, $10 \mathrm{ml}$ each time, 3 times each day, for 7 days.

Evaluation index: At baseline and at 3 and 7 day intervals following treatment, all patients were respectively extracted venous blood for testing the concentration of D-lactate. At the same time, intra-abdominal pressure (IAP) and bowel sounds were recorded. Monitoring of intra-abdominal pressure was conducted by bladder manometry (Wang et al., 2015). The duration of mechanical ventilation, the time of stay in ICU and the mortality rate during 28 days were recorded. Any adverse drug reactions in all enrolled patients during this trial were recorded. 
Statistical analysis: The analysis was determined applying SPSS17.0 software (SPSS software Inc., Chicago, IL, USA). Continuous data was expressed as mean \pm standard deviation; the differences between groups were tested with the Student t-test. Percentage and non-normally distributed data were compared for statistical significance using Chi square-test or the Mann-Whitney U-test. The significance level was established at p <0.05 (two-sided).

\section{Results}

Patient characteristics: No patients were excluded and all 62 subjects entered the statistical analysis. There were no adverse drug reactions in any of these patients during this trial. There were no significant differences in the terms of gender, age, weight, primary disease, APACHE II score between the two groups ( $\mathrm{P}>0.05)$. The mortality rates during 28 days within both groups were $38.71 \%$ and $35.48 \%$, the treatment group was lower than the control group, but the difference was not statistically significant $(\mathrm{P}>0.05)$. The duration of mechanical ventilation and the time of stay in ICU in the treatment group were less than those in the control group $(\mathrm{P}<0.05)$. Refer to Table 1.

Table 1 Demographic characteristics

\begin{tabular}{|c|c|c|c|}
\hline & Control $[n=31]$ & Treatment $[\mathrm{n}=31]$ & $x^{2} / \mathrm{t}, \mathrm{p}$ value \\
\hline Gender $(\mathrm{M} / \mathrm{F}, \mathrm{n})$ & $18 / 13$ & $17 / 14$ & $x^{2}=0.066, \mathrm{p}=0.798$ \\
\hline Age $\left(\overline{\boldsymbol{x}}_{ \pm \mathrm{s}}\right.$, years $)$ & $68.14 \pm 3.76$ & $67.53 \pm 4.01$ & $\mathrm{t}=0.618, \mathrm{p}=0.538$ \\
\hline Weight $\left(\overline{\boldsymbol{x}}_{ \pm \mathrm{s}, \mathrm{kg}}\right)$ & $64.22 \pm 4.61$ & $64.16 \pm 4.36$ & $\mathrm{t}=0.053, \mathrm{p}=0.958$ \\
\hline APACHE II $\left(\overline{\boldsymbol{x}}_{ \pm \mathrm{s}}\right.$, pions $)$ & $19.64 \pm 2.81$ & $19.76 \pm 2.75$ & $\mathrm{t}=0.170, \mathrm{p}=0.865$ \\
\hline Primary disease (n) & & & $x^{2}=1.368, \mathrm{p}=0.968$ \\
\hline Severe pneumonia & 8 & 7 & \\
\hline Pulmonary embolism & 2 & 3 & \\
\hline Chest trauma & 6 & 7 & \\
\hline Cerebrovascular disease & 5 & 6 & \\
\hline Brain injury & 6 & 4 & \\
\hline Poisoning & 1 & 2 & \\
\hline Others & 3 & 2 & \\
\hline Mortality rate (n, \%) & $12(38.71)$ & $11(35.48)$ & $x^{2}=0.69, \mathrm{p}=0.793$ \\
\hline Duration of ventilation $\left(\overline{\boldsymbol{x}}_{ \pm \mathrm{s}}, \mathrm{d}\right)$ & $13.84 \pm 3.51$ & $11.07 \pm 3.16$ & $\mathrm{t}=3.266, \mathrm{p}=0.002$ \\
\hline Time in ICU $\left(\overline{\boldsymbol{x}}_{ \pm \mathrm{s}, \mathrm{d}}\right)$ & $16.59 \pm 2.92$ & $13.81 \pm 2.78$ & $\mathrm{t}=3.839, \mathrm{p}=0.000$ \\
\hline
\end{tabular}

Change of D-lactate: At baseline, there was no statistical difference in the concentrations of D-lactate between the two groups $(\mathrm{P}>0.05)$. At 3 and 7-days following treatment, concentrations of D-lactate recorded in the treatment group were lower than those in control group, and these differences were statistically significant $(\mathrm{P}<0.05)$. Refer to Figure 1. 


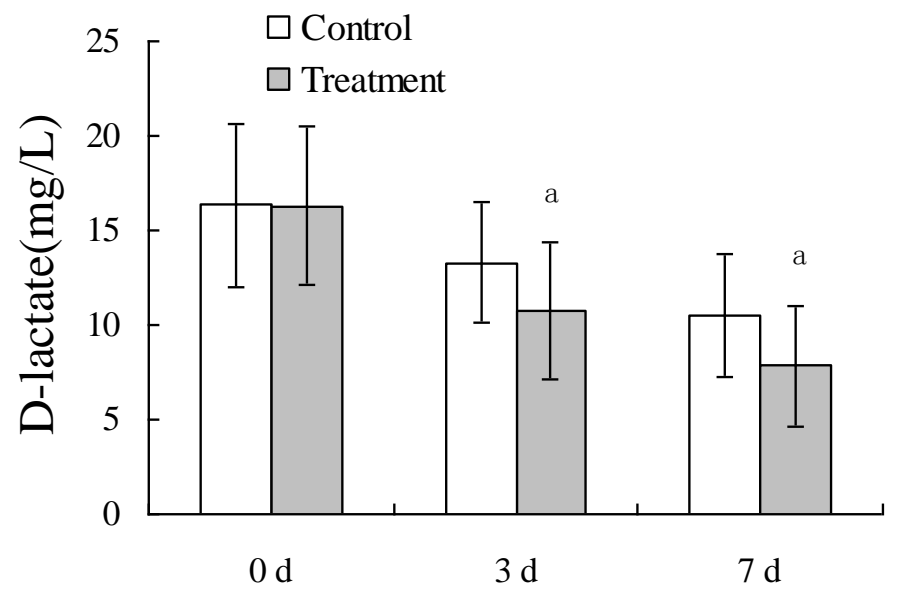

Figure 1 Comparison of D-lactate

Note: All patients received comprehensive treatment. The patients in treatment group additionally received Kangfuxin Liquid, $10 \mathrm{ml}$ Kangfuxin Liquid was administered by naso-gastric tube, 3 times each day, for 7 days. Treatment group compared with control group, ${ }^{\mathrm{a}} \mathrm{P}<0.05$.

Change of IAP: At baseline, there was no statistical difference of the IAP between the two groups (P>0.05). At 3 and 7-days following treatment, the IAP in treatment group was lower than those in the control group and this difference was statistically significant $(\mathrm{P}<0.05)$. Refer to figure 2 .

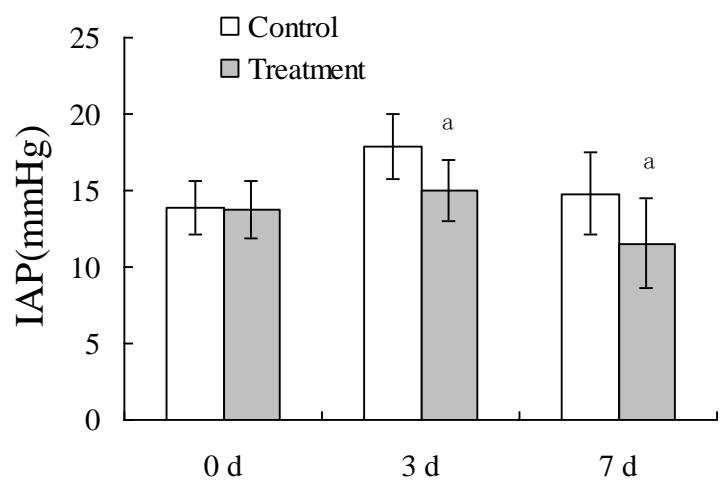

Figure 2 Comparison of intra-abdominal pressure (IAP)

Note: All patients received comprehensive treatment. The patients in treatment group additionally received Kangfuxin Liquid, $10 \mathrm{ml}$ Kangfuxin Liquid administered by naso-gastric tube, 3 times each day, for 7 days. Treatment group compared with control group, ${ }^{\mathrm{a}} P<0.05$.

Change of bowel sounds: At baseline, there was no statistical difference of the bowel sounds between the two groups $(\mathrm{P}>0.05)$. At 3 and 7-days following treatment, the bowel sounds in treatment group were higher than those in control group, and these differences were statistically significant $(\mathrm{P}<0.05)$. Refer to figure 3 . 


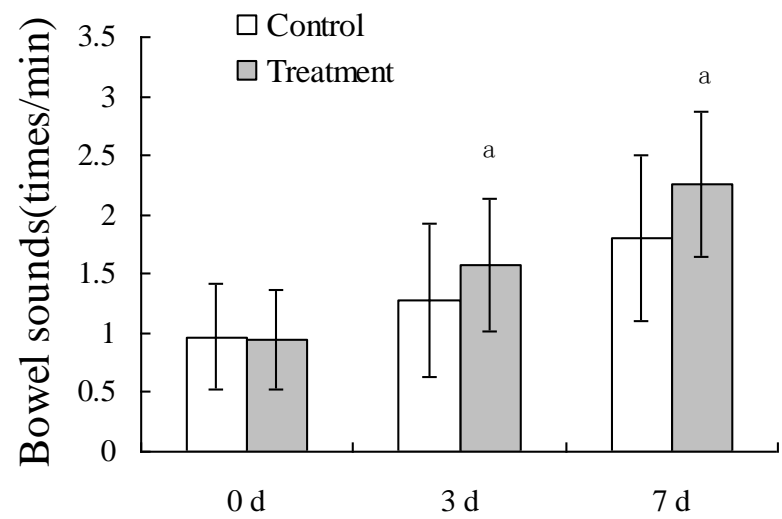

Figure 3 Comparison of bowel sounds

Note: All patients received comprehensive treatment. The patients in treatment group additionally received Kangfuxin Liquid, $10 \mathrm{ml}$ Kangfuxin Liquid was administered by naso-gastric tube, 3 times each day, for 7 days. Treatment group compared with control group, ${ }^{\mathrm{a}} \mathrm{P}<0.05$.

\section{Discussion}

During the process of mechanical ventilation, the work of respiratory muscle lessens and this often leads to gastrointestinal dysfunction (Montejo, 1999; Mutlu et al., 2001). The physiological function in the elderly is degraded, the intestinal function is likewise diminished. The intestinal blood flow is reduced, intestinal blood-vessels shrink and intestinal mucosa is in a low state of profusion during mechanical ventilation. As intestinal mucous damage continues to rise, intestinal mucous barrier function starts weakening. Elderly patients in a state of mechanical ventilation are often fraught with panic, anxiety, depression and other adverse psychological effects. This low mood also leads to increased severe intestinal barrier dysfunction. Moxley (Moxley, 2000) proposed that intestinal barrier dysfunction can lead to intestinal bacteria and toxins being absorbed into the bloodstream and enterogenic infection can occur. This directly causes systemic inflammatory response syndrome (SIRS) or multiple organ dysfunction syndrome (MODS). Gastrointestinal tract is the portal for effective control of MODS, and effective prevention. Treatment of intestinal barrier dysfunction is the key to preventing the occurrence of MODS, which can reduce the mortality rate in critical patients (Rings, 2004). Therefore, it is important that effective intervention on intestinal barrier function in elderly patients having mechanical ventilation in its early stage be applied for controlling the patients' condition.

The increased permeability of intestinal mucosa is an important manifestation of intestinal mucosal barrier dysfunction (Bjarnason et al., 1995). When the hypoperfusion injury of intestinal mucosa occurs, the uppermost epithelium along the intestinal mucosa villus shed and the permeability of intestinal mucosa increases. Surplus D-lactate is then produced by intestinal bacteria entering into the blood stream through the damaged mucosa and the level of D-lactate in plasma is increased. The level of D-lactate is positively correlated with the change of intestinal permeability. D-lactate is a better marker recording intestinal mucosal permeability (Sheng et al., 2015; Welch, 1999). The intestine is very sensitive to any increase in intra-abdominal pressure, as ischemia and hypoxia of intestinal mucosa often leads to intra-abdominal hypertension. Intra-abdominal hypertension can then indirectly affect the severity of intestinal injury (Bodnár et al., 2010). Bowel sounds is an indicator of gastrointestinal peristalsis, which can gauge the recovery of intestinal barrier function from pathological conditions. This marker has the advantages being simple and practical when assessing intestinal barrier function (Zhang et al., 2016a).

Kangfuxin Liquid is a liquid herbal extract obtained from the dried worm of the America periplaneta. It consists of peptide, polyols, epidermal growth factor, sticky sugar amino acids, and other active substances. Latest research 
indicates that Kangfuxin Liquid can reduce the level of endotoxin in patients suffering from sepsis and this enhances the gastrointestinal mucosal barrier function (Zhang et al., 2013). Likewise, it can aid patients suffering with gastrointestinal ulcers because it regulates the ph value of gastric fluid and repairs gastrointestinal mucosal tissue. (Zhang et al., 2012). Other studies support the case that the extract of America periplaneta can adjust the level of immunoglobulin A (IgA), immunoglobulin $\mathrm{G}(\operatorname{IgG})$ and other immune factors and this improves the immune function (Srivastava et al., 2011; Zhang et al., 2016b). In this study, the treatment group given Kangfuxin Liquid, saw D-lactate decreasing, intra-abdominal pressure falling and accompanying bowel sounds increasing at 3 and 7-days following treatment. The changes using these indicators in the "treatment" group were superior than those in the "control" group. These results confirm Kangfuxin Liquid as a protective medicine for intestinal mucosa promoting intestinal peristalsis and enhancing the intestinal barrier function.

Conflict of Interest: The authors declare that they have no competing interests in this study.

\section{Acknowledgements}

Funding: This work was supported by the Science and Technology Research and Development Project of Tangshan City of Hebei Province (NO.15130219a).

Contributors: Zhang HW and Wei LY designed the study, and wrote the first draft. Zhao G provided his advice. Liu $\mathrm{SZ}$ and Zhang $\mathrm{J}$ analyzed the data. All authors approved the final version of the article.

\section{References}

1. Bjarnason, I.; Macpherson, A. and Hollander, D. (1995). Intestinal permeability: an overview. Gastroenterology. 108(5): 1566-1581.

2. Bodnár, Z.; Keresztes, T.; Kovács, I.; Hajdu, Z.; Boissonneault, G.A. and Sipka, S. (2010). Increased serum adenosine and interleukin 10 levels as new laboratory markers of increased intra-abdominal pressure. Langenbeck's Archives of Surgery. 395(7): 969-972.

3. Fang, X.J.; Fang, Q.; Cui, W.; Luo, J.J. and Cai, H.L. (2006). Effects of rude rhubarb on intestinal barrier in septic patients. Chinese Journal of Emergency Medicine. 10(15): 927-929.

4. Geboes, K. and Dalle, I. (2002). Vasculitis and the gastrointestinal tract. Acta gastro-enterologica Belgica. 65(4): 204-212.

5. Montejo, J.C. (1999). Enteral nutrition-related gastrointestinal complications in critically ill patients: a multicenter study: the Nutritional and Metabolic Working Group of the Spanish Society of Intensive Care Medicine and Coronary Units. Critical Care Medicine. 27(8): 1447 -1453.

6. Mutlu, G.M.; Mutlu, E.A. and Factor, P. (2001). GI complications in patients receiving mechanical ventilation. Chest. 119(4):1222-1241.

7. Moxley, R.A. (2000). Edema disease. Veterinary Clinics of North America: Food Animal Practice. 16(1): 175-185.

8. Pan, Y.; Wu, L.Q. and Lu, G.C. (2016). Application of glutamine combined with dietary fibre and fructo-oligosaccharide in enteral nutrition support of elderly patients with mechanical ventilation. Chinese Journal of Geriatrics. 35 (1): 42-45.

9. Qiu, H.C.; Lou, H.L.; Ma, J. and Lin, G.D. (2011). Observation of sijunzi decoction combined with dietary fiber to improve elderly patients with gastrointestinal barrier function. Hebei Medical Journal. 33 (21): 3334-3335.

10. Rings, D.M. (2004). Clostridial disease associated with neurologic signs: tetanus, botulism, and enterotoxemia. Veterinary Clinics of North America: Food Animal Practice. 20(2): 379-391. 
11. Srivastava, D.; Gaur, S.N.; Arora, N. and Singh, B.P. (2011). Clinico-immunological changes post-immunotherapy with Periplaneta americana. European Journal of Clinical Investigation. 41(8): 879-888.

12. Sheng, Y.; Xie, X.H.; Jin, W.J.; Wang, J.E.; Cai, J.F.; Wang, Z.H.; Gao, B. and Zhu, N. (2015). Therapeutic and preventive effects of early drugs intervention on intestinal dysfunction in sepsis patients. Chinese Journal of Critical Care Medicine. 35(4): 304-307.

13. Wang, H.F.; Wang, Y.Q.; Li, Y.; Gao, H.M. and Chang, W.X. (2015). The impact of relevant factors in mechanical ventilation on intra-abdominal pressure in patients with ALI/ARDS. Chinese Journal of Emergency Medicine. 24(12): 1430-1435.

14. Wang, H.G.; Wang, Y. and Guo, Q.M. (2013). Kangfuxin Liquid Combined with pantoprazole in treatment of 120 cases gastric mucosal resection after ulcer associated. Chinese Journal of Integrated Traditional and Western Medicine on Digestion. 21 (7): 382-383.

15. Welch, M. (1999). D-lactate as an early marker of intestinal ischaemia after raptured abdominal aortic aneurysm repair. British Journal of Surgery. 86 (5):712.

16. Zhang, H.; Wei, L.; Zhang, Z.; Liu, S.; Zhao, G.; Zhang, J. and Hu, Y. (2013). Protective effect of periplaneta americana extract on intestinal mucosal barrier function in patients with sepsis. Journal of Traditional Chinese Medicine. 33(1): 70-73.

17. Zhang, H.W.; Wei, L.Y.; Zhang, Z.Y.; Liu, S.Z. and Zhang, J. (2012). Preventive effect of periplaneta americana extract on stress ulcer bleeding in patients with acute lung injury or acute respiratory distress syndrome. Chinese General Practice. 15(33): 3878-3879.

18. Zhang, H.W.; Wei, L.Y.; Zhao, G.; Liu, S.Z.; Yang, Y.J.; Zhang, Z.Y. and Zhang, J. (2016a). Early using periplaneta americana extract on gastrointestinal function in critically patients with infection. Chinese Journal of Primary Medicine and Pharmacy. 23(10): 1456-1458.

19. Zhang, H.W.; Wei, L.Y. Zhao, G. Yang, Y.J.; Liu, S.Z.; Zhang, Z.Y.; Zhang, J. and Hu, Y.L. (2016b). Periplaneta americana extract used in patients with systemic inflammatory response syndrome. World Journal of Emergency Medicine. 7(1): 50-54. 